\title{
Antipsychotic drug use associated with urinary tract infections in older women
}

\author{
Astrid M. van Strien ${ }^{\mathrm{a}, *}$, Patrick C. Souverein ${ }^{\mathrm{b}}$, Carolina (Karen) J.P.W. Keijsers ${ }^{\mathrm{a}}$, \\ Eibert R. Heerdink ${ }^{\mathrm{b}, \mathrm{e}}$, Hieronymus (Jeroen) J. Derijks ${ }^{\mathrm{b}, \mathrm{c}}$, Rob J. van Marum ${ }^{\mathrm{a}, \mathrm{d}}$ \\ a Department of Geriatric Medicine, Jeroen Bosch Hospital, 's-Hertogenbosch, The Netherlands \\ b Utrecht Institute for Pharmaceutical Sciences (UIPS), Division of Pharmacoepidemiology and Clinical Pharmacology, Utrecht University, Utrecht, The \\ Netherlands \\ ${ }^{\mathrm{c}}$ Hospital Pharmacy ZANOB, 's-Hertogenbosch, The Netherlands \\ 'Department of General Practice and Elderly Care Medicine VU University Medical Center, Amsterdam, The Netherlands \\ e Department of Clinical Pharmacy, University Medical Center Utrecht, Utrecht, The Netherlands
}

\section{A R T I C L E I N F O}

\section{Article history:}

Received 16 December 2016

Received in revised form 11 January 2017

Accepted 16 January 2017

\section{Keywords:}

Antipsychotics

Nitrofurantoin

Urinary tract infection

\begin{abstract}
A B S T R A C T
Objectives: Antipsychotic drugs are frequently prescribed to elderly patients, but they are associated with serious adverse effects. The objective of the current study was to investigate the association between use of antipsychotics by elderly women and the risk of urinary tract infections (UTIs).

Cohort study setting: Dispensing data were obtained from the PHARMO Database Network for the period 1998-2008.

Participants: Ambulatory Dutch women ( $\geq 65$ years) with current and past use of antipsychotics.

Measurements: Incidence rates of UTIs, as defined by use of nitrofurantoin, was calculated within and outside the period of exposure to antipsychotic drugs. Cox proportional hazard regression analysis with Andersen-Gill extension for recurrent events was used to calculate crude and adjusted hazard ratios (HRs).

Results: During the study period, 18,541 women with a first prescription of an antipsychotic were identified. Current use of antipsychotics was associated with an increased risk of UTI compared to past use: HR adjusted for age and history of UTIs, 1.33, 95\% CI 1.27-1.39. A strong temporal relationship was found: the risk of being treated for a UTI was higher in the first week after the start of the treatment (adjusted HR 3.03, 95\% CI 2.63-3.50) and decreased after 3 months (adjusted HR 1.22, 95\% CI 1.17-1.28). Cumulative exposure was not associated with an increased risk of UTIs. There was no difference in effect between conventional and atypical antipsychotics.

Conclusion: Our results show an increased risk of uncomplicated UTIs during antipsychotic use in older female patients, especially in the first week of treatment.
\end{abstract}

(c) 2017 Elsevier B.V. All rights reserved.

\section{Introduction}

Antipsychotic drugs are approved for the treatment of schizophrenia and bipolar disorder [1]. While they are frequently prescribed to older patients, atypical antipsychotics are often used outside their approved indication, to treat behavioral disturbances in elderly patients with dementia [1]. A recent study in the United Kingdom reports a rather high prevalence of antipsychotic drug use of $1 \%$ in a primary care setting [2]. Yet, these drugs may cause

* Corresponding author at: Department of Geriatric Medicine, Jeroen Bosch Hospital, PO Box 90153, 5200 ME, 's-Hertogenbosch, The Netherlands.

E-mail address: A.v.Strien@jbz.nl (A.M. van Strien). serious adverse effects. In 2008, the Food and Drug Administration reported that the use of antipsychotics to treat behavioral disorders in elderly patients with dementia was associated with an increased mortality rate [3,4]. Although the cause of this increased mortality is not completely understood, antipsychotic drug use is associated with an increased risk of cardiovascular events, such as stroke, thrombo-embolic events, and cardiac arrhythmia, and infections, such as pneumonia [5]. The risk of bacterial infections was found to be higher in nursing home residents starting conventional antipsychotics than in similar residents starting atypical antipsychotics [6].

Although these drugs increase the risk of bacterial infection, such as pneumonia, it is unclear whether this is also the case for urinary tract infections (UTIs). Urinary tract problems, such as 
incontinence and urine retention, are reported in users of both typical and atypical antipsychotics [7]. These problems may be caused by extrapyramidal side effects, due to anticholinergic side effects or peripheral $\alpha 1$-adrenergic blockade, and may increase the susceptibility to UTIs [7]. UTIs are very common in the elderly population [8]. In The Netherlands, in primary care there are on average 70 episodes of UTIs per 1000 patients-year in women of all ages, with the highest incidence in women $>60$ years old [9].

Since UTIs are a major cause of morbidity and mortality in elderly people and antipsychotic drugs are prescribed frequently to these individuals, an association between these two factors would be clinically relevant. Therefore, the aim of this study was to investigate the association between antipsychotic use in elderly women and the risk of UTIs.

\section{Methods}

\subsection{Design}

This population-based cohort study involved ambulatory Dutch female patients $\geq 65$ years with current and past use of antipsychotics, with or without the occurrence of an uncomplicated UTI.

\subsection{Setting}

Data were obtained from the PHARMO Database Network (Pharmo Institute, Utrecht, The Netherlands; available at: http:// www.pharmo.nl). The PHARMO database network includes the pharmacy dispense records of over 3 million community-dwelling residents in The Netherlands from 1998 onward. Patient information includes gender and date of birth. Because most patients in The Netherlands are registered with a single community pharmacy, records are virtually complete with regard to prescription drugs [10]. The computerized drug-dispense histories contain information about the dispensed drug, dispense date, the prescriber, amount dispensed, and the prescribed dosage regimen. The dispense date is the day the patient or caregiver picked up the prescription at the pharmacy. The duration of use of each dispensed drug can be estimated from the database by dividing the number of dispensed units by the prescribed number of units to be used per day. The database does not provide information about the indications for use of the medication or registration of non-prescription medication. Drugs are coded according to the Anatomical Therapeutic Chemical (ATC) classification. All PHARMO-linked research is in accordance with Dutch privacy and ethical regulations.

\subsection{Participants}

Female patients ( $\geq 65$ years) with at least one prescription of an antipsychotic drug between 1998 and 2008 were identified. Drugs starting with the four-digit ATC code N05A (with the exception of lithium) were classified as antipsychotics. The date of the first antipsychotic prescription marked the start of follow-up. Patients were then followed up until the end of the study period, the censoring date in the database, or death of the patient, whichever came first. All patients were eligible for inclusion if they had one year of prior history in PHARMO before the start of follow-up. The rationale for including female patients only was because we defined our study outcome of uncomplicated UTI on the basis of the use of nitrofurantoin, which is the first-choice drug for treating uncomplicated UTIs in women in The Netherlands, but not in men [9].

\subsection{Exposure definition}

Exposure was defined as the use of antipsychotic drugs. For all patients, we classified follow-up time into periods of current use and past use of antipsychotics. To assess periods of current use, treatment episodes were constructed. Antipsychotic treatment episodes were established by summing consecutive drug deliveries by the pharmacy [11]. If an antipsychotic prescription with the same drug was collected by the patient before the theoretical end date of the previous prescription, the number of overlapping days (units at home) was added to the end date of the subsequent antipsychotic prescription. We allowed for a 14-day permissible gap between the theoretical end date of an antipsychotic prescription and the next one. We created separate treatment episodes for individual antipsychotic initially, and combined these episodes to allow concurrent use of multiple types of antipsychotic drugs. If the duration of a subsequent prescription overlapped that of a subscription for another antipsychotic, the patient was considered to have switched therapy and the remaining tablet days from the first prescription were disregarded. After the end of a treatment episode, patients were classified as past users, until a new treatment episode occurred. We chose past antipsychotic use as reference period, because the patient characteristics were then comparable in both timeframes. To allow for time-dependent updates of covariates e.g. potential confounders, periods of current and past use were split into periods of maximally 182 days. The first 14 days of past use were considered a washout period where no events were counted.

Periods of current use were further stratified according to:

(I) duration (of each current use episode, not cumulatively over follow-up) in 1-7, 8-14, 15-30, 31-90, >90 days;

(II) mean number of standardized defined daily doses (DDD) per day, this is the assumed average maintenance dose per day of a drug used for its main indication in adults and is defined by the World Health Organization (WHO) [12]. For example, the DDD of haloperidol is $8 \mathrm{mg}$ per day for the treatment of psychosis in adults. In general, older patients receive lower doses of antipsychotic medications than younger patients. We used DDD to create comparative doses for different drugs with different potencies. The DDD was categorized into $<0.125$, $0.125-0.5$, and $>0.5$ DDD.

(III) type of antipsychotic: 1) use of atypical antipsychotics (clozapine, olanzapine, quetiapine, tetrabenazine, sulpiride, tiapride, risperidone, aripiprazole); 2) use of conventional antipsychotics (bromperidol, chlorprothixene, droperidol, fluphenazine, flupentixol, fluspirilene, haloperidol, lurasidone, paliperidone, penfluridol, perphenazine, periciazine, pimozide, pipamperone, sertindole, zuclopenthixol); 3) concurrent use of more than one antipsychotic agent.

\subsection{Outcome definition: recurrent events}

The outcome of interest was the occurrence of uncomplicated UTI. Since the Pharmo database used does not contain medical diagnoses in general practice, the prescription of a therapeutic dosage of nitrofurantoin (50 mg 4 times a day or $100 \mathrm{mg} 2$ times a day) was used as proxy for uncomplicated UTI. In general, uncomplicated UTI is the sole indication for nitrofurantoin, except for UTI prophylaxis. If a patient received a second prescription of nitrofurantoin within 7 days after the first prescription ended, this was considered one event (cluster). During the event (cluster of days) the patient was not at risk of a recurrent event. As patients may experience several episodes of UTIs, we assessed the occurrence of recurrent UTIs during the whole follow-up period.

\subsection{Potential confounders}

Known risk factors for UTIs that could potentially confound the relationship between antipsychotic drug use and UTIs are age [13], history of UTIs [13], diabetes mellitus [9], being immune 
compromised [9], stroke [13], urine incontinence [13], cognitive impairment [13], disability in daily living [13], kidney stones, or anomalies of the kidney or urinary tract [9]. Age was added directly to the model as covariate [13]. Proxies were used for some risk factors: prescription of nitrofurantoin in the past year for medical history of UTI [13], use of blood glucose-lowering drugs for diabetes mellitus [9], use of immunosuppressive drugs for increased susceptibility to infection [9], use of alpha-blockers for kidney stones [9], use of urinary antispasmodics e.g. oxybutynin, tolterodine, darifenacin, for incontinence [13], rivastigmine or galantamine for cognitive impairment [13] and distigmine or carbachol for incomplete bladder emptying [9]. For stroke, a hospital diagnosis of stroke was used [13]. No data were available about disability in daily life, or anomalies of the kidney or urinary tract.

\subsection{Data analysis}

Incidence rates for UTIs were calculated as the number of UTIs divided by person-time in current and past exposure periods (reference period). The occurrence of an event (UTI) influences the risk of other events. This means that the analysis of recurrent events is complicated by the dependence of the related events.

Cox proportional hazard regression analysis with Andersen-Gill extension for recurrent events was used to calculate crude and adjusted hazard ratios (HRs) for the association between current use of antipsychotics and risk of recurrent UTI. Patients with maintenance therapy of nitrofurantoin (UTI profylaxis) were excluded from the analysis. Confounders were added sequentially to the model as follows: age, comedications as a proxy for other diagnoses, and stroke as hospital diagnosis. To adjust the model, covariates were included in the final multivariate model if they induced a change in beta coefficient of at least $10 \%$ for the individual covariates. P-values of $<0.05$ were considered to be statistically significant. Data analysis was conducted with STATA SE 14 and IBM SPSS for Windows, version 22 (IBM Inc., New York, NY).
Table 1

Characteristics of study population.

\begin{tabular}{ll}
\hline Characteristic & Number $(\%)(\mathrm{n}=18541)$ \\
\hline Mean age (SD) & $81.9(8.1)$ \\
$65-74$ & $3742(20.2 \%)$ \\
$75-84$ & $7275(39.2 \%)$ \\
$85+$ & $7524(40.6 \%)$ \\
& \\
Comedication (6 months before index date) & $2984(16.1 \%)$ \\
Antidiabetic drugs & $1885(10.2 \%)$ \\
Systemic glucocorticoids & $324(1.8 \%)$ \\
Antidementia drugs & $107(0.6 \%)$ \\
Immunosuppressants & $566(3.1 \%)$ \\
Drugs for urinary frequency and incontinence & $321(1.7 \%)$ \\
Maintenance therapy nitrofurantoin & $47(0.3 \%)$ \\
Distigmine & $144(0.8 \%)$ \\
Alpha-blockers & $2521(13.6 \%)$ \\
History of urinary tract infection & \\
Admission (ever before index date) & $446(2.4 \%)$ \\
Stroke & \\
\hline
\end{tabular}

\section{Results}

During the study period, 18541 women with a first prescription of an antipsychotic drug were identified (mean age at entry into the study 81.9 years, SD 8.1). The characteristics of the study population are displayed in Table 1.

The incidence of UTIs among current antipsychotic users was $29.8 / 100$ person-years versus $20.2 / 100$ person-years in the reference period, during past use, yielding an incidence rate ratio (IRR) 1.47 (95\% CI 1.42-1.54). Using Cox-regression analysis, current use of antipsychotics was associated with an increased risk of UTI. Adjustment for age and history of UTI lowered the magnitude of the effect, but it remained statistically significant. Current use of antipsychotics was associated with a 33\% increased risk of UTIs compared with past use (adjusted HR 1.33, 95\% CI 1.27-1.39). Table 2 shows the results.

The risk of getting a UTI was particularly high in the first week after start of the antipsychotic medication (adjusted HR 3.03,

Table 2

Hazard ratio of uncomplicated urinary tract infections according to nitrofurantoin prescription in female antipsychotic users.

\begin{tabular}{|c|c|c|c|c|c|}
\hline & Number of UTIs & Person years & Crude HR (95\% CI) & Age adjusted HR (95\% CI) & Full adjusted* HR (95\% CI) \\
\hline Past use of antipsychotic & 3913 & 19398 & 1.00 (reference) & 1.00 (reference) & 1.00 (reference) \\
\hline Current use of antipsychotic & 4671 & 15664 & $1.57(1.50-1.63)$ & $1.46(1.39-1.52)$ & $1.33(1.27-1.39)$ \\
\hline \multicolumn{6}{|l|}{$\begin{array}{l}\text { Analysis within current antipsychotic users } \\
\text { Duration of antipsychotic use (days) }\end{array}$} \\
\hline $1-7$ & 201 & 335 & $3.33(2.89-3.84)$ & $3.07(2.66-3.54)$ & $3.03(2.63-3.50)$ \\
\hline $8-14$ & 130 & 307 & $2.34(1.96-2.78)$ & $2.14(1.80-2.55)$ & $2.04(1.71-2.43)$ \\
\hline $15-30$ & 217 & 616 & $1.96(1.70-2.24)$ & $1.78(1.54-2.04)$ & $1.71(1.49-1.96)$ \\
\hline $31-90$ & 613 & 1697 & $2.02(1.85-2.20)$ & $1.83(1.68-1.99)$ & $1.76(1.61-1.91)$ \\
\hline$>90$ & 3509 & 12710 & $1.43(1.37-1.50)$ & $1.34(1.28-1.40)$ & $1.22(1.17-1.28)$ \\
\hline \multicolumn{6}{|l|}{ Defined daily doses of antipsychotic (DDD) ${ }^{\mathrm{a}}$} \\
\hline \multicolumn{6}{|l|}{ Monotherapy atypical } \\
\hline$<0.125$ & 317 & 1064 & $1.41(1.26-1.58)$ & $1.43(1.28-1.61)$ & $1.29(1.15-1.45)$ \\
\hline $0.125-0.5$ & 650 & 2606 & $1.22(1.12-1.32)$ & $1.26(1.16-1.37)$ & $1.20(1.11-1.31)$ \\
\hline$>0.5$ & 134 & 655 & $1.04(0.88-1.24)$ & $1.24(1.04-1.47)$ & $1.15(0.97-1.36)$ \\
\hline \multicolumn{6}{|l|}{ Monotherapy conventional } \\
\hline$<0.125$ & 1470 & 4730 & $1.66(1.57-1.77)$ & $1.42(1.34-1.51)$ & $1.30(1.22-1.38)$ \\
\hline $0.125-0.5$ & 1754 & 5565 & $1.72(1.63-1.82)$ & $1.54(1.46-1.63)$ & $1.40(1.32-1.48)$ \\
\hline$>0.5$ & 127 & 491 & $1.56(1.30-1.86)$ & $1.80(1.51-2.15)$ & $1.59(1.33-1.90)$ \\
\hline \multicolumn{6}{|l|}{ Type of antipsychotics } \\
\hline Atypical antipsychotics ${ }^{\mathrm{b}}$ & 1101 & 4325 & $1.24(1.16-1.33)$ & $1.30(1.22-1.39)$ & $1.22(1.13-1.30)$ \\
\hline Conventional antipsychotics ${ }^{c}$ & 3351 & 10786 & $1.69(1.61-1.77)$ & $1.50(1.43-1.57)$ & $1.36(1.30-1.43)$ \\
\hline Concurrent use of more than one antipsychotic & 219 & 553 & $2.11(1.84-2.42)$ & $1.92(1.67-2.20)$ & $1.67(1.46-1.91)$ \\
\hline
\end{tabular}

$\mathrm{CI}=$ Confidence interval; HR = Hazard ratio; $\mathrm{UTI}=$ urinary tract infection; Full adjusted*: adjusted for age and history of urinary tract infection.

a $\mathrm{DDD}=$ defined daily dose. Defined daily dose of haloperidol for example is $8 \mathrm{mg}$ for treatment of psychosis in adults.

b Clozapine, olanzapine, quetiapine, tetrabenazine, sulpiride, tiapride, risperidone, aripiprazole.

c Phenothiazines, butyrophenones, indoles, thioxanthenes, diphenylbutylamine. 
95\% CI 2.63-3.50) and decreased after 3 months (HR 1.22, 95\% CI 1.17-1.28). The association of atypical antipsychotic drug use with UTI was dose related in a reverse way. The higher the dose of atypical antipsychotics, the lower the risk of UTI. Whereas the cumulative dose of conventional antipsychotics was dose related (adjusted HR 1.30, 95\% CI 1.22-1.38 for DDD <0.25) and (HR 1.59, 95\% CI 1.33-1.90 for DDD >0.5.) There was no difference in effect between conventional and atypical antipsychotics. Conventional antipsychotics showed a slightly higher point estimator (HR 1.36, 95\% CI 1.30-1.43) than atypical antipsychotics (HR $1.22,95 \% \mathrm{CI}$ 1.13-1.30), but $95 \%$ confidence interval was overlapping.

\section{Discussion}

To our knowledge, this is the first study to report an increased risk of UTIs in patients currently using antipsychotics. We showed that the antipsychotic-associated increased risk of UTIs occurred primarily in the first week of treatment. It is possible that these patients had delirium caused by a UTI, so that the relation in these patients would be the other way around, protopathic bias. This is less likely for patients who were prescribed an antipsychotic first and then nitrofurantoin $>7$ days after the start of the antipsychotic. We found UTIs to be associated with both conventional and atypical antipsychotics.

It is unclear whether the observed association between current antipsychotic use and UTIs is related to antipsychotic use or the underlying disease or delirium itself. Theoretically, both could be the case. Older female patients with behavioral disturbances of dementia may be more susceptible to UTIs because of malnutrition, wrong wiping after urination, poor hygiene, or going to the toilet less often. Urination is controlled by a complex mechanism that coordinates bladder storage, emptying, and urinary sphincter activity, by regulating smooth muscle tone in the bladder and urethra [14]. Haloperidol, a conventional antipsychotic, is the first choice antipsychotic for the treatment of delirium in Europe [15]. Conventional antipsychotics like haloperidol are in general stronger D2-receptor antagonists than atypical antipsychotics [14]. D2-receptor antagonists have been suggested to influence the capacity and residual volume of the bladder, external urethral sphincter function, and the relaxation pressure and volume of urine at micturition via inhibition of the spinobulbar reflexes [14]. Also anticholinergic effects of antipsychotics may play a role. The retention of urine caused by these agents can lead to bacterial growth, and UTI's. However, in our sample, the prevalence of antipsychotics with a strong anticholinergic profile (thioridazine, clozapine, chlorpromazine, olanzapine) was very low [16]. The association of antipsychotic drug use and different infections (pneumonia, UTI's) suggests that there is an effect of antipsychotic drugs on the immune system. Psychotropic medications have been shown to modulate immune activation. However, the effects of individual psychotropic agents on the immune system and how these might contribute to their efficacy remain largely unclear [17].

The strengths of this study are its population-based nature, the substantial sample size, and the reliable collection of longitudinal data on antipsychotic and nitrofurantoin prescriptions. However, it also had limitations. The use of a prescription database limited the ability to determine comorbidity except by the proxy of a prescription. As we did not have access to clinical data, the presence of a UTI was based on the prescription of nitrofurantoin, which could have led to misclassification. The Dutch College of General Practitioners (NHG) Clinical guideline Urinary Tract Infections gives nitrofurantoin as the first-choice treatment for uncomplicated UTIs in non-pregnant women [9]. It is possible that there was an overdiagnosis of UTI, particularly if nitrofurantoin was started before confirmatory results of a UTI were available. This is especially relevant to the possibility that an antipsychotic was prescribed for agitation or delirium that was misattributed to a UTI [18]. In general, Dutch physicians are reluctant to prescribe antimicrobial drugs because of the risk of resistance, and treat only those patients with a proven or very high suspicion of infection [19]. For this reason, we think that the likelihood of misclassification is limited. Complicated UTIs are treated with antibiotics that reach urine and tissue, such as fluoroquinolones [9], and so we cannot generalize our findings to complicated UTIs. The association between uncomplicated UTIs and antipsychotic use is probably an underestimation, because antibiotics such as fosfomycine and trimethoprim are also prescribed for uncomplicated UTIs [9].

In conclusion, our results show that the risk of uncomplicated UTIs in older, female users of antipsychotics is increased after medication is started. Clinicians should be alert to the occurrence of UTIs after the start of an antipsychotic drug, especially in the first week. Further research is necessary to confirm these findings. If this is also the case for men using antipsychotics and women with complicated UTIs remains to be established in future studies.

\section{Contributors}

AMvS made substantial contributions to conception and design of the study and was responsible for drafting the article, and was responsible for analysis and interpretation of the data.

PCS made substantial contributions to the conception and design of the study, revised the manuscript for important intellectual content and approved the final version, and was responsible for analysis and interpretation of the data.

CJPWK made substantial contributions to the conception and design of the study, revised the manuscript for important intellectual content and approved the final version.

ERH made substantial contributions to the conception and design of the study, revised the manuscript for important intellectual content and approved the final version.

HJD made substantial contributions to the conception and design of the study, revised the manuscript for important intellectual content and approved the final version.

RJvM made substantial contributions to the conception and design of the study, revised the manuscript for important intellectual content and approved the final version.

\section{Conflict of interest}

The authors declare that they have no conflict of interest.

\section{Funding}

No funding was received for this study/article.

\section{Ethical approval}

All PHARMO-linked research is in accordance with Dutch privacy and ethical regulations.

\section{Provenance and peer review}

This article has undergone peer review.

\section{References}

[1] S.A. Hollingworth, D.J. Siskind, L.M. Nissen, et al., Patterns of antipsychotic medication use in Australia 2002-2007, Aust. N. Z. J. Psychiatry 44 (2010) $372-377$

[2] L. Marston, I. Nazareth, I. Petersen, et al., Prescribing of antipsychotics in UK primary care: a cohort study, BMJ Open 4 (2014) e006135, 2014-006135. 
[3] FDA, Deaths with Antipsychotics in Elderly Patients with Behavioral Disturbances, Department of Health and Human Services, Washington, DC, 2005, Available at: http://www.fda.gov/drugs/drugsafety/ postmarketdrugsafetyinformationforpatientsandproviders/ucm053171. (Accessed December 2016).

[4] FDA, Deaths with Antipsychotics in Elderly Patients with Behavioral Disturbances, Conventional Antipsychotics, Department of Health and Human Services, Washington, DC, 2008, Available at: http://www.fda.gov/Drugs/ DrugSafety/PostmarketDrugSafetyInformationforPatientsandProviders/ ucm124830. htm. (Accessed December 2016).

[5] W. Knol, R.J. van Marum, P.A. Jansen, et al., Antipsychotic drug use and risk of pneumonia in elderly people, J. Am. Geriatr. Soc. 56 (2008) 661-666.

[6] K.F. Huybrechts, S. Schneeweiss, T. Gerhard, et al., Comparative safety of antipsychotic medications in nursing home residents, J. Am. Geriatr. Soc. 60 (2012) 420-429.

[7] S. Saddichha, M. Kumar, Antipsychotic-induced urinary dysfunction: anticholinergic effect or otherwise? BMJ Case Rep. 2009 (2009), http://dx.doi. org/10.1136/bcr.02.2009.1547, Epub 2009 May 21.

[8] S.J. Matthews, J.W. Lancaster, Urinary tract infections in the elderly population, Am. J. Geriatr. Pharmacother. 9 (2011) 286-309.

[9] Dutch College of General Practitioners (NHG), Clinical Guideline (M05): Urinary Tract Infections, 2013, Available at: https://www.nhg.org/ standaarden/samenvatting/urineweginfecties. (Accessed December 2016).

[10] H. Buurma, M.L. Bouvy, P.A. De Smet, et al., Prevalence and determinants of pharmacy shopping behaviour, J. Clin. Pharm. Ther. 33 (2008) 17-23.
[11] H. Gardarsdottir, P.C. Souverein, T.C. Egberts, et al., Construction of drug treatment episodes from drug-dispensing histories is influenced by the gap length, J. Clin. Epidemiol. 63 (2010) 422-427.

[12] WHO Collaborating Center, Definition of DDD Defined Daily Dose. Available at: http://www.whocc.no/ddd/definition_and_general_considera/. (Accessed December 2016).

[13] M.A. Caljouw, W.P. den Elzen, H.J. Cools, et al., Predictive factors of urinary tract infections among the oldest old in the general population. A population-based prospective follow-up study, BMC Med. 9 (57) (2011), 7015-9-57.

[14] N. Faure Walker, K. Brinchmann, D. Batura, Linking the evidence between urinary retention and antipsychotic or antidepressant drugs: a systematic review, Neurourol. Urodyn. (2015).

[15] A. Morandi, D. Davis, J.K. Taylor, et al., Consensus and variations in opinions on delirium care: a survey of European delirium specialists, Int. Psychogeriatr. 25 (2013) 2067-2075.

[16] M.L. Chew, B.H. Mulsant, B.G. Pollock, et al., Anticholinergic activity of 107 medications commonly used by older adults, J. Am. Geriatr. Soc. 56 (2008) 1333-1341.

[17] D. Baumeister, S. Ciufolini, V. Mondelli, Effects of psychotropic drugs on inflammation: consequence or mediator of therapeutic effects in psychiatric treatment? Psychopharmacology (Berl.) 233 (2016) 1575-1589.

[18] H.J. Woodford, J. George, Diagnosis and management of urinary tract infection in hospitalized older people, J. Am. Geriatr. Soc. 57 (2009) 107-114.

[19] N. van de Sande-Bruinsma, H. Grundmann, D. Verloo, et al., Antimicrobial drug use and resistance in Europe, Emerg. Infect. Dis. 14 (2008) 1722-1730. 